\title{
Residential Locale Is Associated with Disparities in Genetic Testing-Related Outcomes Among BRCA1/2-Positive Women
}

\author{
Kate E. Dibble ${ }^{1}\left[\right.$ D $\cdot$ Avonne E. Connor ${ }^{1,2}$
}

Received: 1 November 2021 / Revised: 4 February 2022 / Accepted: 7 February 2022 / Published online: 17 February 2022

(c) W. Montague Cobb-NMA Health Institute 2022

\begin{abstract}
Background While research on hereditary genetic testing for BRCA1/2 mutations continues to emerge, there remain unanswered questions regarding access to testing and cancer-related care. Our study determined the associations between race/ ethnicity, residential locale, and genetic testing provider and related outcomes among US women with BRCAl/2 genetic mutations.

Methods One hundred ninety-three BRCA1/2-positive women from vulnerable health backgrounds were recruited via private national Facebook BRCA1/2-oriented support groups and completed an online survey. Adjusted odds ratios (aOR) and $95 \%$ confidence intervals (CIs) were estimated using multivariable logistic regression for the associations between race/ethnicity, residential locale, and genetic testing-related outcomes.

Results Women ranged in age $(18-75, M=39.5, S D=10.7)$, and most were non-Hispanic white $(66.3 \%)$ and lived in a suburban locale (54.9\%). Women living in suburban areas were significantly less likely (aOR, .369, 95\% CI, .177-.771) to receive behavioral referrals after genetic testing compared to those living in an urban locale. Women living in rural areas and suburban areas were 4.72 times more likely $(95 \%$ CI, 1.48-15.1, $p=.009)$ and 2.61 times more likely (95\% CI, 1.05-6.48, $p=.038$ ), respectively, to receive genetic testing from a primary care provider versus private genetic testing office/hospital compared to women in urban locales. Associations between race/ethnicity and genetic testing outcomes were not statistically significant. Residential locale did not predict the odds of undergoing surgery for risk reduction or surveillance for early detection.

Conclusion Our study identifies disparities in genetic testing resources among women living in suburban and rural areas. These findings can be used to inform future care, research, and community resources that may impact services relating to genetic testing within these locales.
\end{abstract}

Keywords Residential disparities $\cdot$ Racial/ethnic disparities $\cdot$ Genetic testing $\cdot$ Health access $\cdot$ Breast cancer

$\begin{array}{ll}\text { Abbreviations } \\ \text { BRCA } & \text { Breast cancer } \\ \text { DTC } & \text { Direct-to-consumer } \\ \text { NHW } & \text { Non-Hispanic white } \\ \text { PCP } & \text { Primary care provider } \\ \text { US } & \text { United States }\end{array}$

Kate E. Dibble

kdibble2@jhu.edu

1 Department of Epidemiology, Johns Hopkins Bloomberg School of Public Health, 615 N. Wolfe Street, Baltimore, MD 21205, USA

2 Department of Oncology, Johns Hopkins Sidney Kimmel Comprehensive Cancer Center, Baltimore, MD 21205, USA

\section{Introduction}

One in eight women in the USA will be diagnosed with breast cancer in their lifetime; however, among these women, 5-10\% test positive for BRCA1 and/or BRCA2 (breast cancer) genetic mutations [1,2]. Although rare, these mutations occur on dominant genes and are indicative of a 50\% chance to inherit and, therefore, reside within biological family units. These genes are damaged, allowing breast, ovarian, and other associated cancers to grow more quickly than among those without these mutations [3, 4]. The presence of these mutations may result in an increase in breast and ovarian cancer risk, up to $72 \%$ and $44 \%$ for $B R C A 1$, in addition to 69 to $17 \%$ increased risk for those with $B R C A 2$ before the age of 70 , respectively [5, 6]. Despite the increases in screening and prophylactic surgery, being 
diagnosed with a $B R C A 1 / 2$ mutation post-cancer diagnosis has been associated with higher risks of mortality [7], as those with $B R C A 1$ may be more likely to be diagnosed with triple-negative breast cancer, while $B R C A 2$-positive women may be more likely to have hormone-receptor positive tumors [6]. Rates of recurrence among cancer survivors with $B R C A 1 / 2$ mutations remain high without prophylactic surgery. Female cancer survivors with these mutations remain approximately 1.5 times more likely than women without these mutations to experience a cancer recurrence $[8,9]$; however, this association is dependent on hormone receptor status [10], treatment(s), stage at diagnosis [6], and relative lifestyle factors such as alcohol use, current and past weight, and smoking, among others [11]. Prophylactic surgery for risk reduction (e.g., hysterectomy, bilateral mastectomy, salpingectomy, oophorectomy) is a widely used option to reduce breast and ovarian cancer risk among BRCA1/2positive women $[12,13]$. Women who are diagnosed with $B R C A 1 / 2$ mutations prior to a cancer diagnosis and undergo prophylactic surgery have lower incidence of related cancers [14]. Ongoing surveillance methods (e.g., self-examination, magnetic resonance imaging [MRI], transvaginal ultrasound, mammogram, bloodwork) are utilized among women who are planning to or are currently having children, possibly in combination with partial prophylactic surgery or chemoprevention $[15,16]$.

Genetic testing-related outcomes for BRCA1/2-positive women from vulnerable health populations [17-19], such as racial/ethnic minorities, those identifying as lesbian, gay, transgender, queer/questioning, or others (LGBTQ+), those living in poverty, first-generation immigrants, and women with chronic conditions or physical disabilities, are relatively unknown. Previous research has focused heavily on groups that have higher prevalence of $B R C A 1 / 2$ mutations, such as Ashkenazi Jewish women, due to the presence of a founder mutation, a specific gene mutation founded by a small group of ancestors geographically (or culturally) isolated [20]. The prevalence of $B R C A 1 / 2$ mutations among other racial or ethnic groups has been noted. In a data repository study conducted by Myriad Genetics, BRCA1/2 mutations were found in $14.8 \%, 15.6 \%, 12.7 \%$, and $13.2 \%$ of tested Hispanic, African, Asian, and Native American women, respectively. Previous literature has focused heavily on the psychosocial impact of receiving a BRCA1/2 diagnosis [21-23] and undergoing surgery or surveillance for risk reduction or early detection [24-26]. Unfortunately, most of these studies have sampled high-risk English-speaking non-Hispanic white women [22, 25, 27], such as Ashkenazi Jewish populations [28-30], limiting the generalizability that genetic testing and BRCAl/2-related experiences have to racial/ethnic groups. Despite this impact, behavioral referrals are uncommon [27]. These referrals, in combination with the impact of ongoing surveillance and prophylactic surgeries, are studied qualitatively, again due to the rarity and difficulty of recruiting BRCA1/2-positive women [27]. These types of studies also focus primarily on women at high risk like those with cancer histories and/or Ashkenazi Jewish heritage.

In recent years, genetic testing has become readily available from a variety of sources, including but not limited to genetic testing offices, hospital programs, primary care providers (PCPs), specialists (e.g., oncologists, gynecologists), and via direct-to-consumer (DTC) tests (e.g., 23andMe $\odot$, Jax $\odot$, etc.) [31-34]. There also exist two types of genetic counseling in the USA that are offered across the genetic testing sources listed above. Individualized testing solely tests the individual within the family identified to be at greatest risk for having a genetic mutation, whereas familybased testing involves all at-risk family members undergoing testing and receiving results together as a group [35]. Although both testing types exist in the USA, individualized testing remains more accessible and affordable, as familybased testing is not offered everywhere and is not covered by most health insurance plans [36]. According to the American Medical Association (AMA) [37], providers who order genetic testing should (A) have appropriate knowledge and expertise to counsel patients on heritable conditions, (B) adhere to standards of non-directive counseling, and (C) discuss family history, proposed genetic tests, and medical and psychological implications for the patient and biological relatives. However, genetic testing and genome knowledge necessary to comprehensively order and explain results of such testing remains low among certain populations including providers at pivotal junctures such as gynecologists and PCPs, allowing miscommunication and misinformation to occur [38-42].

It is evident that disparities exist among breast and ovarian cancer prevention and care among those with BRCA1/2 mutations from vulnerable health populations [43]. Specifically, non-Hispanic white (NHW) women living above the federal poverty level are more likely to be tested for BRCA1/2 mutations compared to racial/ethnic minorities because these women are more likely to have access to quality healthcare and have the financial means to complete testing $[44,45]$. Disparities also exist in hereditary genetic testing due to residential locale, where geographical differences exist across the USA. One such study found that BRCA1/2-positive women with employer-sponsored health insurance living in western and northeastern USA are more likely to undergo genetic testing than women in southern or midwestern US [46]. Findings also suggest that those living in a metropolitan area are more likely to receive an MRI or mammogram within a year of $B R C A 1 / 2$ diagnosis compared to those living in a non-metropolitan area [46]. While research on BRCA1/2 mutations continues to emerge, there are questions regarding genetic testing outcomes and how they relate to disparate groups, such as racial/ethnic 
minorities and those with limited physical access to quality genetic testing. Therefore, the impact of race/ethnicity and residential locale remains vague among this population, as past studies have faced recruitment challenges because of BRCA1/2 rarity [47, 48]. Few studies have incorporated numerous vulnerable health populations with these genetic mutations, such as those described in the current study, to determine their likelihood of undergoing risk-reducing surgery or ongoing surveillance, as previous literature briefly suggested [2]. Furthermore, access to genetic counselors and dearth of genetic testing-related follow-up care have not been widely studied distinctly among disparate groups [47].

Objectives The current study aims to expand on past literature by highlighting the likelihood of women from racial/ ethnic minority backgrounds and those living in specific residential locales report the following: behavioral referral, type of genetic testing, being genetically tested via a PCP versus other locations/methods, and surgery or ongoing surveillance. Women at-risk for $B R C A 1 / 2$ mutations may experience barriers to quality genetic testing and ongoing care, and we hypothesize that racial/ethnic minority women and women living in rural and suburban locales may have more challenges in receiving $B R C A 1 / 2$-related care.

\section{Materials and Methods}

\section{Study Design and Sample}

Women were recruited through national, online private support groups: BRCA1 BRCA2 Genetic Ovarian and Breast Cancer Gene ( 11,000 members), BRCA Genetic Sisters Support Group ( 6000 members), BRCA1 \& BRCA2 Support Group ( 3300 members), BRCA Strong ( 2500 members), BRCA Sisterhood of Hope ( 1400 members), Understanding BRCA ( 1500 members) BRCA Advanced \& Other Hereditary Cancers Journal Club ( 3,200 members), BRCA Preventive Mastectomy \& Hysterectomy Support Group ( 900 members), and Facing Our Risk of Cancer Empowered (FORCE) [49] forums. Written permission to join each group and post for research purposes was obtained via group moderators prior to posting. Recruitment opened in December 2020 and ended when the recruitment ceiling was reached in March 2021 at 225 consented participants. Once recruitment post was posted per week within each Facebook and forum page except for BRCA Strong group, who allowed one post biweekly. Recruitment posts consisted of a brief announcement introducing the nature of the study, eligibility criteria, link to anonymous survey and the survey passcode. Eligibility was restricted to (1) women 18 or older; (2) living in the USA; (3) who could read and speak English; (4) those who had tested positive for BRCAI and/or $B R C A 2$ within the past 5 years; and (5) identify with at least one vulnerable health population (racial or ethnic minority, those identifying as LGBTQ +, person with a physical disability, those with low income, first-generation immigrant, and/or those with chronic conditions). The presence of chronic conditions were determined based upon the question, "Have you ever been diagnosed with the following by a physician?", based on the Charlson Comorbidity Index [50]. The responses included Alzheimer's disease or dementia, arthritis, asthma, chronic pulmonary disease, genetic immune disease [lupus, multiple sclerosis, etc.], diabetes, heart disease, hypertension, deficiency anemias, obesity, hypothyroidism, depression, HIV/AIDS, epilepsy, Parkinson's disease, or other, with the possibility to select multiple options. Potential participants clicked the survey link from the recruitment post and were rerouted to an anonymous screener survey to determine eligibility. If fitting the above criteria, participants were rerouted once more and asked to enter the survey passcode, allowing them to access the full online survey hosted by REDCap (Research Electronic Data Capture) [51, 52] managed by Johns Hopkins Bloomberg School of Public Health (JHSPH) information technology (IT) department. Participants provided consent by electronically signing a consent form via REDCap prior to beginning the survey, which consisted of sociodemographic characteristics, clinical cancer and genetic testing information, prophylactic surgery and ongoing surveillance history, healthcare discrimination, healthcare access, and self-reported psychosocial measures. Although 225 women were eligible and consented, 211 completed the online survey. Missingness was handled by excluding participants casewise as the final analytic sample was 193. Participants were compensated with a \$20 Amazon e-gift card upon completion of the online survey. This study was reviewed and approved according to the ethical standards of the JHSPH Institutional Review Board (IRB).

\section{Model Variables}

Predictor variables In the current analyses, two self-reported predictors (race/ethnicity, residential locale) were utilized. Race and ethnicity were originally two separate variables. Race was categorical (American Indian or Alaska Native, Asian, Native Hawaiian or other Pacific Islander, Black or African American, non-Hispanic white [NHW], biracial or multiracial), and ethnicity was dichotomous (not Hispanic or Latino, Hispanic or Latino). These variables were combined to create a racial/ethnic dichotomous variable (NHW, racial/ ethnic minority). Residential locale remained categorical (urban area [e.g., densely populated cities], suburban area [e.g., populated areas outside city limits], and rural area [e.g., far outside city limits, woods, and farmland]). 
Outcome assessments The current study analyzed five self-reported genetic testing-related outcomes: (1) behavioral referral offered post-testing, (2) type of genetic testing received, (3) location of genetic testing, (4) prophylactic surgery for risk reduction, and (5) ongoing surveillance for early detection.

Two outcome variables were originally categorical: behavioral referral offered post-testing (no; yes, offered for those with positive results; yes, offered for everyone) and location of genetic testing (private genetic testing office; hospital; primary care physician [PCP]; direct-to-consumer

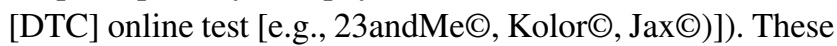
variables were dichotomized for the purposes of analyses: behavioral referral offered post-testing (affirmative responses [referent], no) and location of genetic testing (private genetic testing office or hospital [referent], PCP). For the model of testing location, DTC cases $(n=8)$ were excluded but were included in all other models. Type of genetic testing (individualized [referent], family-based), surgery for risk reduction (yes [referent], no surgeries), and ongoing surveillance for early detection (yes [referent], no surveillance) remained dichotomous.

Covariates Covariates were entered across all models to ensure comparability: age at survey completion, years since genetic testing, out-of-pocket cost for genetic testing, education, marital status, poverty status, and cancer history due to previous literature support $[1,5,19,53]$. Predictors were also mutually adjusted. Age at survey completion, years since genetic testing, and out-of-pocket cost for genetic testing were treated as continuous. Several categorical variables were categorical such as education (less than high school, high school graduate or GED, some college or technical school, college graduate, some graduate school, master's degree, professional degree [JD, MD], doctoral degree) and marital status (married/living as married, divorced, separated, widowed, single) and were condensed into dichotomous variables for ease of interpretation: education (some college or less [referent], college graduate or above) and marital status (married/living as married [referent], other). Annual household income was originally categorical, ranging from less than $\$ 20,000$ to $\$ 200,000$ or more, and was dichotomized by creating a cutoff pertaining to poverty status per household (less than $\$ 40,000$ annually [referent], $\$ 40,000$ annually or more) based on the US Census Bureau [54]. Cancer history (no cancer history [referent], cancer history) remained dichotomous.

\section{Statistical Methods}

Analyses were conducted utilizing Stata statistical software, version 16 [55]. Univariate chi-square tests for categorical variables and independent samples $t$-tests for continuous variables, combined with analyses of variance, were conducted to determine potential covariates for sociodemographic characteristics of interest. Crude (ORs) and adjusted odds ratios (aORs) with $95 \%$ confidence intervals (CIs) were estimated using multivariable logistic regression models to determine an association between predictors (race/ethnicity, residential locale) and genetic testing-related outcomes while adjusting for potential confounders. All statistical tests were two-sided, and statistical significance was indicated if $p$-values were below 0.05 .

\section{Results}

\section{Characteristics of the Sample}

Characteristics of the study population, overall and stratified by racial/ethnic minority status and residential locale, are presented in Table 1. A total of 193 BRCA1/2-positive women, both with and without a history of breast and/or ovarian cancer meeting eligibility criteria, were included in analyses. Women ranged in age from 18 to 75 years $(M=39.5, S D=10.7)$, most of whom did not have a history of either cancer $(n=133,61.7 \%)$. Most women were NHW ( $n=128,66.3 \%)$, completed a college or graduate degree $(n=125,64.8 \%)$, not impoverished $(n=150,77.7 \%)$, and lived in a suburban locale $(n=106,54.9 \%)$. Per outcome, most women reported not being offered behavioral referral post-testing ( $n=124,64.2 \%)$, completed individualized genetic testing ( $n=170,88.1 \%)$, underwent prophylactic surgeries for risk reduction $(n=129,66.8 \%)$, and/or ongoing surveillance for early detection $(n=174,90.2 \%)$. Results showed that private genetic testing offices were utilized less often in rural locales compared with urban and suburban locales. Type of genetic mutation was split, with $43.1 \%$ diagnosed with $B R C A 1$ and $56.9 \%$ with $B R C A 2$ mutations. NHW women more often received family-based genetic testing $(p=0.005)$ and were older at survey completion $(p=0.040)$ than racial/ethnic minority women. Women living in urban locales paid significantly more for genetic testing than those living in rural locales $(p=0.000)$.

\section{Genetic Testing-Related Outcomes by Race/Ethnicity and Residential Locale}

The associations between race/ethnicity, residential locale, and the odds of receiving a behavioral referral after testing positive for a $B R C A 1 / 2$ mutation versus not receiving a referral are presented in Table 2. Women living in suburban locales (e.g., populated areas outside city limits) were significantly less likely (OR, $0.463,95 \%$ CI, 0.239-0.897, $p=0.023$ ) to report being referred for behavioral therapy after testing positive for a BRCAl/2 mutation compared to 
Table 1 Participant demographic characteristics, overall and by racial/ethnic minority status

\begin{tabular}{|c|c|c|c|c|c|c|c|c|}
\hline & \multicolumn{8}{|l|}{ Predictors } \\
\hline & NHW $(n=128)$ & $\begin{array}{l}\text { Racial/ethnic } \\
\text { minority } \\
(n=65)\end{array}$ & $p$-value & $\begin{array}{l}\text { Subur- } \\
\text { ban locale } \\
(n=106)\end{array}$ & $\begin{array}{l}\text { Urban locale } \\
(n=58)\end{array}$ & $\begin{array}{l}\text { Rural locale } \\
(n=29)\end{array}$ & $p$-value & $\begin{array}{l}\text { Total analytic } \\
\text { sample } \\
(N=193)\end{array}$ \\
\hline & No. $(\%)$ & No. $(\%)$ & & No. $(\%)$ & No. $(\%)$ & No. $(\%)$ & & No. $(\%)$ \\
\hline \multicolumn{9}{|c|}{ Vulnerable health population characteristics } \\
\hline \multicolumn{9}{|l|}{ Disability status } \\
\hline No disability & $87(68.0)$ & $49(75.4)$ & 0.286 & $41(70.7)$ & $75(70.8)$ & $20(69.0)$ & 0.982 & $136(70.5)$ \\
\hline Disability & $41(32.0)$ & $16(24.6)$ & & $17(29.3)$ & $31(29.3)$ & $9(31.0)$ & & $57(29.5)$ \\
\hline \multicolumn{9}{|l|}{ Sexual orientation } \\
\hline Straight or not gay & $97(75.8)$ & $54(83.1)$ & 0.246 & $45(77.6)$ & $81(76.4)$ & $25(86.2)$ & 0.521 & $151(78.2)$ \\
\hline $\begin{array}{l}\text { LGBTQ + or something } \\
\text { else }\end{array}$ & $31(24.2)$ & $11(16.9)$ & & $13(22.4)$ & $25(23.6)$ & $4(13.8)$ & & $42(21.8)$ \\
\hline \multicolumn{9}{|l|}{ Immigration status } \\
\hline $\begin{array}{l}\text { Not a first-generation } \\
\text { immigrant }\end{array}$ & $122(95.3)$ & $60(92.3)$ & 0.395 & $55(94.8)$ & $99(93.4)$ & $28(96.6)$ & 0.793 & $182(94.3)$ \\
\hline First-generation immigrant & $6(4.7)$ & $5(7.7)$ & & $3(5.2)$ & $7(6.6)$ & $1(3.4)$ & & $11(5.7)$ \\
\hline \multicolumn{9}{|l|}{ Multimorbidity } \\
\hline No multimorbidity & $48(37.5)$ & $28(43.1)$ & 0.454 & $27(46.5)$ & $37(34.9)$ & $12(41.4)$ & 0.335 & $76(39.4)$ \\
\hline $\begin{array}{l}\text { Two or more comorbid } \\
\text { conditions }\end{array}$ & $80(62.5)$ & $37(56.9)$ & & $31(53.5)$ & $69(65.1)$ & 17 (58.6) & & $117(60.6)$ \\
\hline \multicolumn{9}{|l|}{ Covariates } \\
\hline \multicolumn{9}{|l|}{ Education } \\
\hline College degree or above & $78(60.9)$ & $47(72.3)$ & 0.118 & $34(58.6)$ & $69(65.1)$ & $22(75.9)$ & 0.282 & $125(64.8)$ \\
\hline No college degree & $50(39.1)$ & $18(27.7)$ & & $24(31.4)$ & 37 (34.9) & $7(24.1)$ & & $68(35.2)$ \\
\hline \multicolumn{9}{|l|}{ Marital status } \\
\hline $\begin{array}{l}\text { Married or living as mar- } \\
\text { ried }\end{array}$ & $77(60.2)$ & $42(64.6)$ & 0.547 & $33(56.9)$ & $65(61.3)$ & $21(72.4)$ & 0.371 & $119(61.7)$ \\
\hline Other & $51(39.8)$ & $23(35.4)$ & & $25(43.1)$ & $41(38.7)$ & $8(27.6)$ & & $74(38.3)$ \\
\hline \multicolumn{9}{|l|}{ Poverty status } \\
\hline No poverty & $95(74.2)$ & $55(84.6)$ & 0.101 & $39(67.2)$ & $87(82.1)$ & $24(82.8)$ & 0.072 & $150(77.7)$ \\
\hline Poverty & $33(25.8)$ & $10(15.4)$ & & $19(32.8)$ & $19(17.9)$ & $5(17.2)$ & & $43(22.3)$ \\
\hline \multicolumn{9}{|l|}{ Cancer history } \\
\hline No cancer history & $88(68.8)$ & $45(69.2)$ & 0.946 & $44(75.9)$ & $67(63.2)$ & $22(75.9)$ & 0.168 & $133(61.7)$ \\
\hline Cancer history/survivor & $40(31.2)$ & $20(30.8)$ & & $14(24.1)$ & $39(36.8)$ & $7(24.1)$ & & $60(38.3)$ \\
\hline \multicolumn{9}{|l|}{ Outcomes } \\
\hline \multicolumn{9}{|c|}{ Behavioral referral offered post-testing } \\
\hline Affirmative & $46(35.9)$ & $23(35.4)$ & 0.940 & $28(42.3)$ & $32(30.2)$ & $9(31.0)$ & 0.059 & $69(35.8)$ \\
\hline No & $82(64.1)$ & $42(64.6)$ & & $30(51.7)$ & $74(69.8)$ & $20(69.0)$ & & $124(64.2)$ \\
\hline \multicolumn{9}{|c|}{ Type of genetic testing received } \\
\hline Individualized & $108(84.4)$ & $62(95.4)$ & 0.026 & $53(91.4)$ & $91(85.9)$ & $26(89.7)$ & 0.556 & $170(88.1)$ \\
\hline Family-based & $20(15.6)$ & $3(4.6)$ & & $5(8.6)$ & $15(14.1)$ & $3(10.3)$ & & $23(11.9)$ \\
\hline \multicolumn{9}{|l|}{ Location of genetic testing } \\
\hline $\begin{array}{l}\text { Private genetic testing } \\
\text { office }\end{array}$ & $37(28.9)$ & $22(33.9)$ & 0.574 & $21(36.2)$ & $32(30.2)$ & $6(20.7)$ & 0.360 & 59 (30.6) \\
\hline Hospital & $49(38.3)$ & $28(43.1)$ & & $25(43.1)$ & $41(38.7)$ & $11(37.9)$ & & $77(39.9)$ \\
\hline PCP & $37(28.9)$ & $13(20.0)$ & & $9(15.5)$ & $30(28.3)$ & $11(37.9)$ & & $50(25.9)$ \\
\hline DTC & $5(3.9)$ & $2(3.0)$ & & $3(5.2)$ & $3(2.8)$ & $1(3.5)$ & & $7(3.6)$ \\
\hline \multicolumn{9}{|l|}{ Surgery for risk reduction } \\
\hline Yes & $88(68.8)$ & $41(63.1)$ & 0.429 & $33(56.9)$ & $74(69.8)$ & $22(75.9)$ & 0.130 & $129(66.8)$ \\
\hline No & $40(31.2)$ & $24(36.9)$ & & $25(43.1)$ & $32(30.2)$ & $7(24.1)$ & & $64(33.2)$ \\
\hline
\end{tabular}


Table 1 (continued)

\begin{tabular}{|c|c|c|c|c|c|c|c|c|}
\hline & \multicolumn{8}{|l|}{ Predictors } \\
\hline & NHW $(n=128)$ & $\begin{array}{l}\text { Racial/ethnic } \\
\text { minority } \\
(n=65)\end{array}$ & $p$-value & $\begin{array}{l}\text { Subur- } \\
\text { ban locale } \\
(n=106)\end{array}$ & $\begin{array}{l}\text { Urban locale } \\
(n=58)\end{array}$ & $\begin{array}{l}\text { Rural locale } \\
(n=29)\end{array}$ & $p$-value & $\begin{array}{l}\text { Total analytic } \\
\text { sample } \\
(N=193)\end{array}$ \\
\hline & No. (\%) & No. $(\%)$ & & No. (\%) & No. (\%) & No. (\%) & & No. (\%) \\
\hline \multicolumn{9}{|c|}{ Surveillance for early detection } \\
\hline Yes & $115(89.8)$ & $59(90.8)$ & 0.838 & $54(93.1)$ & $94(88.7)$ & $26(89.7)$ & 0.658 & $174(90.2)$ \\
\hline \multirow[t]{2}{*}{ No } & $13(10.2)$ & $6(9.2)$ & & $4(6.9)$ & $12(11.3)$ & $3(10.3)$ & & $19(9.8)$ \\
\hline & Mean $(S D)$ & Mean $(S D)$ & $p$-value & Mean $(S D)$ & Mean $(S D)$ & Mean $(S D)$ & $p$-value & Mean $(S D)$ \\
\hline Age at survey completion & $40.6(11.8)$ & $37.3(8.02)$ & 0.040 & $38.1(10.3)$ & $39.7(11.2)$ & $41.5(9.70)$ & 0.571 & $39.5(10.7)$ \\
\hline Years since genetic testing & $1.95(1.54)$ & $1.83(1.67)$ & 0.637 & $2.06(1.66)$ & $1.90(1.60)$ & $1.63(1.38)$ & 0.530 & $19.1(1.58)$ \\
\hline $\begin{array}{l}\text { Out-of-pocket cost for } \\
\text { genetic testing }\end{array}$ & $383.5(1357.2)$ & $556.6(180.6)$ & 0.179 & $\begin{array}{l}1000.3 \\
(4116.6)\end{array}$ & $\begin{array}{l}374.9 \\
\quad(1350.9)\end{array}$ & $\begin{array}{l}333.6 \\
\quad(1117.8)\end{array}$ & 0.000 & $556.6(2509.3)$ \\
\hline
\end{tabular}

DTC $=$ direct-to-consumer test (e.g., 23andMe@, Kolor@, Jax®, etc.)

LGBTQ + = lesbian, gay, bisexual, transgender, queer/questioning, and all other gender identities and sexual orientations

$\mathrm{NHW}=$ non-Hispanic white

$\mathrm{PCP}=$ primary care provider

*Poverty status $=$ annual household income less than $\$ 40,000$ USD

${ }^{\ddagger}$ Multimorbidity is defined as having two or more comorbid conditions, including any cancer diagnosis

Bolded font indicates significant $p$-value $(<0.05)$

those living in urban locales. After adjusting for covariates, this relationship remained statistically significant (aOR, $0.369,95 \%$ CI, $0.177-0.771, p=0.008$ ). Race/ethnicity was not a significant predictor for odds of receiving a behavioral referral upon a positive $B R C A 1 / 2$ genetic test.

The associations between race/ethnicity, residential locale, and the odds of receiving family-based genetic testing for $B R C A 1 / 2$ mutations compared to individualized genetic testing are presented in Table 3 . In unadjusted models, race/ethnicity was the only significant predictor of receiving family-based genetic testing for BRCAl/2 mutations, where women of minority race/ethnicities were significantly less likely (OR, 0.261, 95\% CI, 0.074-0.914, $p=0.036$ ) to report receiving this type of genetic testing compared to NHW women. This relationship was not significant after adjusting for covariates (aOR, $0.283,95 \% \mathrm{CI}$, $0.071-1.12, p=0.073$ ).

The associations between race/ethnicity, residential locale, and the odds of receiving genetic testing for $B R C A 1 / 2$ mutations from a private genetic testing office or hospital versus PCP are presented in Table 4. In crude models, women from rural locales were 2.87 times more likely to utilize a PCP for genetic testing $(95 \%$ CI, 1.04-7.91, $p=0.041$ ) compared to women in urban locales, and in adjusted models, this association was stronger (aOR, 4.72, 95\% CI, 1.47-15.1, $p=0.009$ ). In adjusted models, women from suburban locales also were more likely to receive genetic testing from PCPs (aOR, $2.61,95 \% \mathrm{CI}, 1.05-6.48, p=0.038$ ) compared to women living in urban locales. Race/ethnicity was not a significant predictor for odds of receiving genetic testing from a PCP for BRCA1/2 mutations.

Race/ethnicity and residential locale were not significant predictors for the odds of being diagnosed with undergoing prophylactic surgery for cancer risk reduction (Supplement Table A) or undergoing ongoing surveillance for cancer early detection (Supplement Table B).

\section{Discussion}

Our study examined the relationship between race/ethnicity, residential locale, and several genetic testing-related outcomes among BRCA1/2-positive women from vulnerable health populations to identify high-risk groups of mutation carriers. We identified issues in genetic testing access in rural and suburban locales, as women living in these areas were significantly more likely to report utilizing a PCP for genetic testing in comparison to women living in urban areas. Interestingly, women living in suburban areas were significantly less likely to receive behavioral referrals after testing positive for BRCA1/2 mutations compared to those living in urban areas. Overall, associations between race/ethnicity and genetic testing outcomes were not statistically significant. To our knowledge, there have not been any other studies focusing on BRCA1/2-related genetic testing outcomes recruited from several vulnerable health populations like delineated within the current study. 
Table 2 Crude and adjusted model odds ratios and $95 \%$ confidence intervals for odds of receiving a behavioral referral after testing positive for BRCA1/2 mutations associated with racial/ethnic minority status and residential locale among $B R C A 1 / 2$-positive women from vulnerable health populations $(N=193)$
Table 3 Crude and adjusted model odds ratios and 95\% confidence intervals for odds of receiving family-based genetic testing associated with racial/ethnic minority status and residential locale among BRCA1/2-positive women from vulnerable health populations $(N=193)$

\begin{tabular}{llllllll}
\hline Predictors & \multicolumn{2}{l}{ Crude model } & & \multicolumn{2}{l}{ Adjusted model } \\
\cline { 3 - 4 } & OR & $95 \%$ CI & $p$-value & & aOR & $95 \%$ CI & $p$-value \\
\hline Race/ethnicity & 1.00 & Referent & & & 1.00 & Referent & \\
NHW & 0.976 & $0.523-1.82$ & 0.940 & & 0.894 & $0.442-1.80$ & 0.755 \\
Minority race/ethnicity & & & & & & & \\
Residential locale & 1.00 & Referent & & & 1.00 & Referent & \\
Urban & 0.463 & $0.239-0.897$ & $\mathbf{0 . 0 2 3}$ & & 0.369 & $0.177-0.771$ & $\mathbf{0 . 0 0 8}$ \\
Suburban & 0.482 & $0.188-1.23$ & 0.128 & & 0.400 & $0.139-1.14$ & 0.088 \\
\hline Rural & & & & & &
\end{tabular}

$\mathrm{OR}=$ odds ratio

$\mathrm{aOR}=$ adjusted odds ratio

$\mathrm{CI}=$ confidence interval

$\mathrm{NHW}=$ non-Hispanic white

Bold font indicates statistically significant with corresponding $p<0.05$

Covariates: age at questionnaire (continuous), years since genetic testing (continuous), out-of-pocket cost for genetic testing (continuous), education (some college or less, college graduate or above), marital status (married or living as married, other), poverty status (above $\$ 40,000$ annually, below $\$ 40,001$ annually), and cancer history (no cancer history, cancer history)

Predictors: race/ethnicity (NHW [referent], minority race/ethnicity [includes American Indian/Alaska Native, Asian, Native Hawaiian/Other Pacific Islander, Black/African American, biracial or multiracial, Hispanic]) and residential locale (urban [referent], suburban, rural). Predictors are also mutually adjusted

\begin{tabular}{llllllll}
\hline Predictors & \multicolumn{2}{l}{ Crude model } & & \multicolumn{2}{l}{ Adjusted model } \\
& OR & $95 \%$ CI & $p$-value & & aOR & $95 \%$ CI & $p$-value \\
\hline Race/ethnicity & 1.00 & Referent & & & 1.00 & Referent & \\
NHW & 0.261 & $0.074-0.914$ & $\mathbf{0 . 0 3 6}$ & & 0.283 & $0.071-1.12$ & 0.073 \\
Minority race/ethnicity & & & & & & & \\
Residential locale & 1.00 & Referent & & & 1.00 & Referent & \\
Urban & 1.74 & $0.600-5.08$ & 0.305 & & 1.88 & $0.578-6.12$ & 0.293 \\
Suburban & 1.22 & $0.271-5.51$ & 0.793 & & 1.05 & $0.201-5.53$ & 0.949 \\
Rural & & & & & & &
\end{tabular}

$\mathrm{OR}=$ odds ratio

$\mathrm{aOR}=$ adjusted odds ratio

$\mathrm{CI}=$ confidence interval

NHW $=$ non-Hispanic white

Bold font indicates statistically significant with corresponding $p<0.05$

Covariates: age at questionnaire (continuous), years since genetic testing (continuous), out-of-pocket cost for genetic testing (continuous), education (some college or less, college graduate or above), marital status (married or living as married, other), poverty status (above $\$ 40,000$ annually, below $\$ 40,001$ annually), and cancer history (no cancer history, cancer history)

Predictors: race/ethnicity (NHW [referent], minority race/ethnicity [includes American Indian/Alaska Native, Asian, Native Hawaiian/Other Pacific Islander, Black/African American, biracial or multiracial, Hispanic]) and residential locale (urban [referent], suburban, rural). Predictors are also mutually adjusted
These findings add to the growing body of literature highlighting apparent issues in access to genetic testing by residential locale experienced by BRCA1/2-positive women.

Due to the increasing availability of genetic testing through various sources (e.g., PCPs, gynecologists, genetic counselors), more patients are completing genetic testing [31-34]. With physical genetic testing offices giving way to telehealth and telemedicine for genetic testing [56], access for those in suburban and rural locales, like those described in this study, is readily available, saving individuals the time 
Table 4 Crude and adjusted model odds ratios and $95 \%$ confidence intervals for odds of receiving genetic testing from a primary care physician (PCP) associated with racial/ethnic minority status and residential locale among BRCA1/2-positive women from vulnerable health populations $(N=185)$

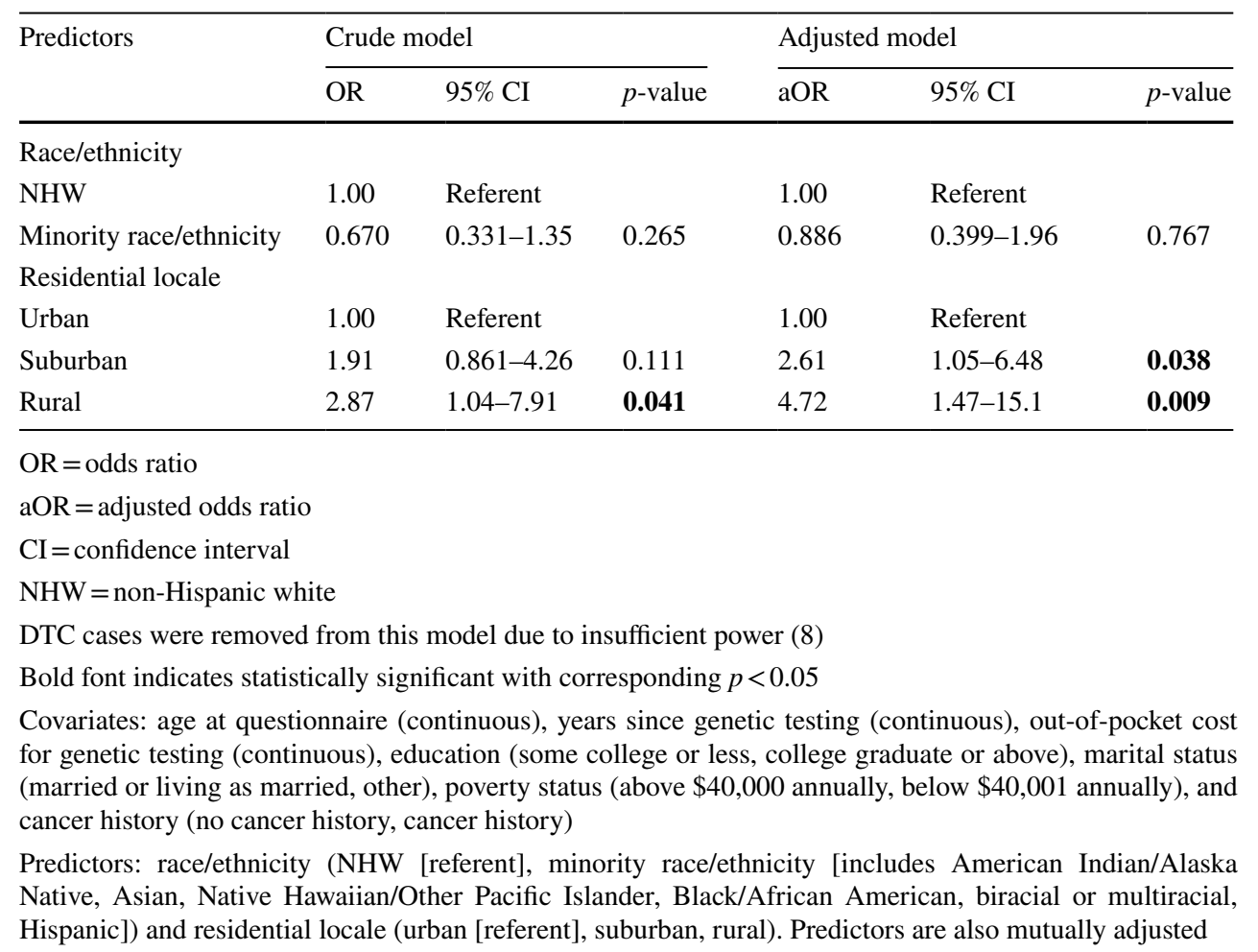

and cost of traveling to a specialist [57]. Cost and physical access remain one of the most limiting factors of where women at risk for $B R C A 1 / 2$ mutations will receive care [57], especially pertinent among rural populations. Associated resources, such as receiving a behavioral referral due to positive genetic testing results, may also be linked with physical access and health insurance coverage among rural locales [57, 58]. Suburban BRCA1/2-positive women were less likely to receive a behavioral referral compared to urban patients, which has not been widely discussed in previous literature. Despite this, research has briefly suggested that other factors among suburban populations may impact the likelihood of receiving a referral and possibly indicative of less referrals, including doctor-patient communication, scheduling flexibility, and already having access to behavioral therapy [58]. Despite the increasing availability of genetic testing both through telehealth and through in-person locations [31-34], past literature has found differences relating to quality of care [59], provider-patient communication $[60,61]$, and applicable resources for those testing positive [62].

Our study findings suggested a possible racial/ethnic disparity in odds of receiving family-based genetic testing prior to adjusting for covariates. It is possible that the field of family-based genetic testing is novel and not widely implemented, as well as having limited research exploring these disparities [19, 63]. Family-based genetic testing is rarely covered by current health insurance plans, even if deemed at-risk, as individualized genetic testing is more accessible and accepted. Lastly, only $32.7 \%$ of our sample identified as a racial/ethnic minority, so a more racially and ethnically diverse sample may have allowed for more robust findings. Literature over the past decade has begun to focus on genetic testing and incidence of BRCA1/2-related cancers among at-risk populations [64]. However, opportunities to refer and test high-risk women from disparate groups such as racial/ ethnic minorities are often missed [65]; only $20-30 \%$ of minority women at risk for breast cancer underwent genetic testing, with these estimates lower among those at risk for ovarian cancer [63]. Family-based genetic testing is not the norm and has been offered less often than individualized genetic testing, at least in the USA [56, 63], and thus, very little research has explored this approach, where the entire family unit identified at risk for genetic mutations undergoes genetic testing together $[66,67]$. This could perhaps be due to the way healthcare insurance reimburses for services, which bases medical visits per individual, not per family unit. Generational differences and geographical restraints regarding genetic testing usability and value, for instance, may also be a reason for low implementation of the familybased approach. Previous literature has noted several barriers to family-based genetic testing and at-risk identification for family members generally, including but not limited to financial, logistical, ethical, legal, social, and clinical challenges associated with such care [63, 68, 69]. Previous literature has also suggested best routes of implementing 
family-based genetic testing via patient preference, providing traceback programs that notify family members [63], adapting genetic testing for vulnerable health and minority populations such as through a universal genetic testing initiative [56], and boosting familial communication and disclosure of genetic test results [70]. We hypothesized that there would be significant associations between racial/ethnic minority status and genetic testing-related outcomes; however, our non-significant findings could be due to the small sample of racial/ethnic minority women. These associations should be examined in larger study samples of racially/ethnically diverse study populations.

Study strengths The current study has several strengths. To our knowledge, the current study is the first to report the percentage of BRCA1/2-positive women who received (or did not receive) a post-genetic testing behavioral referral in the USA. Our study successfully recruited from a combination of hard-to-reach populations in addition to those with hereditary breast/ovarian cancer mutations, which are not easily recruited in-person due to rarity [71]. This study was a pilot and tested recruitment success among BRCA1/2positive women from several vulnerable health populations across the US utilizing online support groups. Past literature has utilized small sample sizes and/or use qualitative methods; however, we were successful in recruiting a moderately large sample of these at-risk women. This study was one of the first, to our knowledge, that recruited BRCA1/2-positive women from vulnerable health populations from across the USA, allowing usually limited generalizability to subgroups such as racial/ethnic minorities, those with low income, and those with cancer histories. Future research can utilize this piloted approach to recruit $B R C A 1 / 2$ women or other hardto-reach groups for rare or stigmatized health conditions in the USA.

Limitations The current study's results should be interpreted considering its limitations. Although the current study successfully recruited a moderately large sample, this study was a cross-sectional study, and data were based on self-report which could lead to misclassification and/or recall bias. Findings should be replicated within larger and even more diverse study samples to confirm trends explained within this manuscript, especially among racial/ethnic minority women. Future research should also include non-English speakers instead of excluding these women from study eligibility. Support groups were utilized as our primary source of recruitment, which have been found to introduce bias by hosting individuals shown to be more open and willing to share private experiences than individuals not in support groups [72]. Therefore, generalization of results and implications are limited to $B R C A 1 / 2$-positive women within various vulnerable health populations in the USA reflected in the current study.

This study provided a unique approach to understanding the impact of residential locale and race/ethnicity on several genetic testing-related outcomes among women with BRCA1/2 mutations from vulnerable health populations. Our findings indicated that there were issues in genetic testing access and associated behavioral referrals among women living in suburban and rural areas of the USA. Future research should purposefully target differences in genetic testing access by geolocation or type of provider (e.g., PCPs, gynecologists, oncologists, genetic counselors, etc.) in a larger sample of BRCA1/2-positive women. Moreover, future longitudinal studies of women at high risk for BRCA1/2 mutations could be conducted to follow their genetic testing process (e.g., prior to testing and afterward) to inform future interventions and care policy for medical providers working with this population. Clinically, medical providers ordering genetic testing for patients should follow AMA recommendations [37] and could supplement their knowledge with additional training and resources to better care for their patients. With genetic testing becoming more widely available, especially via the implementation and increased use of telemedicine in response to the COVID-19 pandemic, it is possible for women at risk for these mutations to receive affordable and quality genetic testing from knowledgeable providers.

Supplementary Information The online version contains supplementary material available at https://doi.org/10.1007/s40615-022-01259-w.

Funding The current study was funded by the Johns Hopkins HoChing Yang Memorial Faculty Award. Dr. Kate Dibble received research support from the National Cancer Institute (T32CA009314) Cancer Epidemiology, Prevention, and Control training program.

Data Availability The datasets generated during and/or analyzed the current study are available at the principal investigator's (PI) discretion upon reasonable request.

Code Availability Syntax coding is available upon reasonable request from the corresponding author.

\section{Declarations}

Consent to Participate Informed consent was obtained from all individual participants included in the study.

Consent for Publication Not applicable.

Competing Interests The authors declare no competing interests. 


\section{References}

1. American Cancer Society (ACS). Family cancer syndromes. 2020. Accessed 18 Jan 2022. https://www.cancer.org/cancer/cancercauses/genetics/family-cancer-syndromes.html.

2. Hall MJ, Reid JE, Burbidge LA, et al. BRCA1 and BRCA2 mutations in women of different ethnicities undergoing testing for hereditary breast-ovarian cancer. Cancer. 2009;115(10):2222-33. https://doi.org/10.1002/cncr.24200.

3. Godet I, Gilkes DM. BRCA1 and BRCA2 mutations and treatment strategies for breast cancer. Integr Cancer Sci Ther. 2017; 4(1): 10. https://doi.org/10.15761/ICST.1000228.

4. Mersch J, Jackson MA, Park M, et al. Cancers associated with BRCA1 and BRCA2 mutations other than breast and ovarian. Cancer. 2015;121(14):269-75. https://doi.org/10.1002/cncr. 29357.

5. Kuchenbaecker KB, Hopper JL, Barnes DR, et al. Risks of breast, ovarian, and contralateral breast cancer for BRCA1 and BRCA2 mutation carriers. JAMA. 2017;317(23):2402-16. https://doi.org/ 10.1001/jama.2017.7112.

6. Song Y, Barry WT, Seah DS, et al. Patterns of recurrence and metasasis in BRCA1/BRCA2-associated breast cancers. Cancer. 2020;126(2):271-80. https://doi.org/10.1002/cncr.32540.

7. Haque R, Shi JM, Telford C, et al. Survival outcomes in BRCA1 or BRCA2 mutation carriers and the influence of triple-negative breast cancer subtype. Perm J. 2018;22:170-97. https://doi.org/ 10.7812/TPP/17-197.

8. Domchek SM, Friebel TM, Singer CF, et al. Association of riskreducing surgery in BRCA1 or BRCA2 mutation carriers with cancer risk and mortality. JAMA. 2010;304(9):967-75. https:// doi.org/10.1001/jama.2010.1237.

9. Torres-Mejía G, Royer R, Llacuachaqui M, et al. Recurrent BRCA1 and BRCA2 mutations in Mexican women with breast cancer. Cancer Epidemiol Biomark Prev. 2015;24(3):498-505. https://doi.org/10.1158/1055-9965.epi-13-0980.

10. Schmidt MMK, van den Broek AJ, Tollenaar RAEM, et al. Breast cancer survival of BRCA1/2 carriers compared to non-BRCA1/2 carriers in a large breast cancer cohort. In: Am Assoc Cancer Res (AACR), 2013. Washington, DC.

11. Yadav S, Ladkany R, Yadav D, et al. Impact of BRCA mutation status on survival of women with triple-negative breast cancer. Clin Breast Cancer. 2018;18(5):e1229-35. https://doi.org/10. 1016/j.clbc.2017.12.014.

12. Lafourcade A, His M, Baglietto L, et al. Factors associated with breast cancer recurrences or mortality and dynamic prediction of death using history of cancer recurrences: the French E3N cohort. BMC Cancer. 2018;18(1):171. https://doi.org/10.1186/ s12885-018-4076-4.

13. Mau C, Untch M. Prophylactic surgery: For whom, when and how. Breast Care. 2017;12:379-84. https://doi.org/10.1159/ 000485830

14. Ludwig KK, Neuner J, Butler A, Geurts JL, Kong AL. Risk reduction and survival benefit of prophylactic surgery in BRCA mutation carriers, a systematic review. Am J Surg. 2016;212(4):660-9. https://doi.org/10.1016/j.amjsurg.2016.06.010.

15. van Zelst JCM, Mus RDM, Woldringh G, et al. Surveillance of women with the BRCA1 or BRCA2 mutation by using biannual automated breast US, MR imaging, and mammography. Radiology. 2017;285(2):376-88. https://doi.org/10.1148/radiol.20171 61218.

16. Nanez A, Stram DA, Garcia C, Powell CB. Ovarian cancer surveillance in the clinical follow up of women with known BRCA1 or BRCA2 pathogenic variants in a large health care system. Gynecol Oncol. in press. https://doi.org/10.1016/j.ygyno.2021.07.034
17. American Journal of Managed Care (AJMC). 5 vulnerable populations in healthcare. 2018. Accessed 18 Jan 2022. https://www. ajmc.com/view/5-vulnerable-populations-in-healthcare.

18. National Institute on Minority Health and Health Disparities (NIMHD). Health disparity populations. 2021. Accessed 18 Jan 2022. https://www.nimhd.nih.gov/about/overview/.

19. Olaya W, Esquivel P, Wong JH, et al. Disparities in BRCA testing: when insurance coverage is not a barrier. Am J Surg. 2009;198(4):562-5. https://doi.org/10.1016/j.amjsurg.2009.07. 003.

20. Centers for Disease Control and Prevention (CDC). The BRCA1 and BRCA2 gene. 2022. Accessed 12 Oct 2021. https://www.cdc. gov/genomics/disease/breast_ovarian_cancer/genes_hboc.htm.

21. Boghosian T, McCuaig JM, Carlsson L, Metcalfe KA. Psychosocial interventions for women with a BRCA1 or BRCA2 mutation: a scoping review. Cancers. 2021;13(7):1486. https://doi.org/10. 3390/cancers 13071486.

22. Glassey R, O'Connor M, Ives A, et al. Heightened perception of breast cancer risk in young women at risk of familial breast cancer. Fam Cancer. 2018;17:15-22. https://doi.org/10.1007/ s10689-017-0001-2.

23. Ringwald J, Wochnowski C, Bosse K, et al. Psychological distress, anxiety, and depression of cancer-affected BRCA1/2 mutation carriers: a systematic review. J Genet Couns. 2016;25(5):880-91. https://doi.org/10.1007/s10897-016-9949-6.

24. Gopie JP, Vasen HFA, Tibben A. Surveillance for hereditary cancer: does the benefit outweigh the psychological burden? A systematic review. Crit Rev Oncol Hematol. 2012;83(3):329-40. https://doi.org/10.1016/j.critrevonc.2012.01.004.

25. Borreani C, Manoukian S, Bianchi E, et al. The psychological impact of breast and ovarian cancer preventive options in BRCA1 and BRCA2 mutation carriers. Clin Genet. 2013;85(1):7-15. https://doi.org/10.1111/cge.12298.

26. Harmsen MG, Hermens RPMG, Prins JB, Hoogerbrugge N, de Hullu JA. How medical choices influence quality of life of women carrying a BRCA mutation. Crit Rev Oncol Hematol. 2015;96(3):555-68. https://doi.org/10.1016/j.critrevonc.2015. 07.010 .

27. Vadaparampil ST, Pickard Quinn G, Brzoscowicz J, Miree CA. Experiences of genetic counseling for BRCA1/2 among recently diagnosed breast cancer patients: a qualitative inquiry. J Psychosoc Oncol. 2008;26(4):33-52. https://doi.org/10.1080/07347 330802359586.

28 Yuen J, Cousens N, Barlow-Stewart K, O'Shea R, Andrews L. Online BRCA1/2 screening in the Australian Jewish community: a qualitative study. J Comm Genet. 2020;11(3):291-302. https:// doi.org/10.1007/s12687-019-00450-7.

29. Trivedi MS, Colbeth H, Yi H, et al. Understanding factors associated with uptake of BRCA1/2 genetic testing among Orthodox Jewish women in the USA using a mixed-methods approach. Public Health Genom. 2018;21:186-96. https://doi.org/10.1159/ 000499852.

30. Nielsen SM, De Simone LM, Olopade OI. Cancer susceptibility genetic testing in a high-risk cohort of urban Ashkenazi Jewish individuals. J Genet Couns. 2018;27(6):1405-10. https://doi.org/ 10.1007/s10897-018-0269-x.

31. Bellcross CA, Peipins LA, McCarty FA, et al. Characteristics associated with genetic counseling referral and BRCA1/2 testing among women in a large integrated health system. Genet Med. 2015;17(1):43-50. https://doi.org/10.1038/gim.2014.68.

32. McCarthy AM, Bristol M, Fredricks T, et al. Are physician recommendations for BRCA $1 / 2$ testing in patients with breast cancer appropriate? A population-based study. Cancer. 2013;119(20):3596-603. https://doi.org/10.1002/cncr.28268.

33. Tutty E, Petelin L, McKinley J, et al. Evaluation of telephone genetic counselling to facilitate germline BRCA1/2 testing in 
women with high-grade serous ovarian cancer. Eur J Hum Genet. 2019;27:1186-96. https://doi.org/10.1038/s41431-019-0390-9.

34. Kinney AY, Steffen LE, Brumbach BH, et al. Randomized noninferiority trial of telephone delivery of BRCA1/2 genetic counseling compared with in-person counseling: 1-year follow-up. J Clin Oncol. 2016;34(24):2914-24. https://doi.org/10.1200/jco. 2015.65.9557.

35. Katapodi MC, Northouse LL, Milliron KJ, et al. Individual and family characteristics associated with BRCA1/2 genetic testing in high-risk families. Psycho-Oncol. 2013;22(6):1336-43. https:// doi.org/10.1002/pon.3139.

36. Frey MK, Kahn RM, Chapman-Davis E, et al. Prospective feasibility trial of a novel strategy of facilitated cascade genetic testing using telephone counseling. J Clin Oncol. 2020;38(13):1389-97. https://doi.org/10.1200/jco.19.02005.

37. American Medical Association (AMA). Genetic testing and counseling. 2021. Accessed 16 Sep 2021. https://www.ama-assn.org/ delivering-care/ethics/genetic-testing-counseling.

38. Haga SB, Kim E, Myers RA, Ginsburg GS. Primary care physicians' knowledge, attitudes, and experience with personal genetic testing. J Pers Med. 2019;9(2):29. https://doi.org/10.3390/jpm90 20029.

39. Hamilton JG, Abdiwahab E, Edwards HM, Fang ML, Jdayani A, Breslau ES. Primary care providers' cancer genetic testing-related knowledge, attitudes, and communication behaviors: a systematic review and research agenda. J Gen Intern Med. 2017;32(3):31524. https://doi.org/10.1007/s11606-016-3943-4.

40. Pace LE, Tung N, Lee YS, et al. Challenges and opportunities in engaging primary care providers in BRCA testing: results from the BFOR study. J Gen Intern Med. 2021; in press. https://doi.org/ 10.1007/s11606-021-06970-8.

41. Grignol VP, Agnese DM. Clinical genetics for the gynecologist. In: Knaus J, Jachtorowycz M, Adajar A, Tam T, editors. Ambulatory Gynecology. Springer; 2018. https://doi.org/10.1007/978-14939-7641-6_19.

42. Carroll JC, Morrison S, Miller FA, Wilson BJ, Permaul JA, Allanson $\mathrm{J}$. Anticipating the primary care role in genomic medicine: expectations of genetics health professionals. J Comm Genet. 2021; in press. https://doi.org/10.1007/s12687-021-00544-1.

43. Cannioto R. Investigating contributions of physical inactivity and obesity to racial disparities in cancer risk and mortality warrants more consideration. J Natl Cancer Inst. 2020;113(6):647-9. https://doi.org/10.1093/jnci/djaa189.

44. Williams CD, Bullard AJ, O'Leary M, Thomas R, Redding TS IV, Goldstein K. Racial/ethnic disparities in BRCA counseling and testing: A narrative review. J Racial Ethn Health Disparities. 2019;6(3):570-83. https://doi.org/10.1007/s40615-018-00556-7.

45. McCarthy AM, Bristol M, Domchek SM, et al. Health care segregation, physician recommendation, and racial disparities in BRCA $1 / 2$ testing among women with breast cancer. J Clin Oncol. 34(22): 2610-2618. https://doi.org/10.1200/JCO.2015.66.0019.

46. Kolor K, Chen Z, Grosse SD, et al. BRCA genetic testing and receipt of preventive interventions among women aged 18-64 years with employer-sponsored health insurance in nonmetropolitan and metropolitan areas - United States 2009-2014. MMWR - Surveill Summ. 2017; 66(15): 1-11. https://doi.org/10.15585/ mmwr.ss6615a1.

47. Jones T, Trivedi MS, Jiang X, et al. Racial and ethnic differences in BRCA1/2 and multigene panel testing among young breast cancer patients. J Cancer Educ. 2019;36(3):463-9. https://doi.org/10. 1007/s13187-019-01646-8.

48. Lieberman S, Tomer A, Ben-Chetrit A, et al. Population screening for BRCA1/BRCA2 founder mutations in Ashkenazi Jews: proactive recruitment compared with self-referral. Genet Med. 2017;19:754-62. https://doi.org/10.1038/gim.2016.182.
49. Facing Our Risk of Cancer Empowered (FORCE.org). Visit our message boards. 2021. Accessed 20 Sep 2021. https://www.facin gourrisk.org/support/message-boards.

50. Charlson ME, Pompei P, Ales KL, MacKenzie CR. A new method of classifying prognostic comorbidity in longitudinal studies: development and validation. J Chron Dis. 1987;40(5):373-83. https://doi.org/10.1016/0021-9681(87)90171-8.

51. Harris PA, Taylor R, Minor BL, et al. The REDCap consortium: building an international community of software platform partners. J Biomed Inform. 2019;95: 103208. https://doi.org/10. 1016/j.jbi.2019.103208.

52. Harris PA, Taylor R, Thielke R, Payne J, Gonzalez N, Conde JG. Research electronic data capture (REDCap) - a metadata-driven methodology and workflow process for providing translational research informatics support. J Biomed Inform. 2009;42(2):37781. https://doi.org/10.1016/j.jbi.2008.08.010.

53. Bayraktar S, Arun BK. BRCA mutation genetic testing implications in the United States. The Breast. 2017;31:224-32. https:// doi.org/10.1016/j.breast.2016.11.021.

54. US Census Bureau. How the Census Bureau measures poverty. 2021. Accessed 10 Nov 2021. https://www.census.gov/topics/ income-poverty/poverty/guidance/poverty-measures.html.

55. StataCorp, Inc. Stata statistical software: release 16. 2019: College Station, TX.

56. Cohen SA, Bradbury A, Henderson V, Hoskins K, Bednar E, Arun BK. Genetic counseling and testing in a community setting: quality, access, and efficiency. Am Soc Clin Oncol Educ Book. 2019;39:34-44. https://doi.org/10.1200/edbk_238937.

57. Rhoads S, Rakes AL. Telehealth technology: Reducing barriers for rural residents seeking genetic counseling. J Am Acad Nurse Pract. 2020;32(3):190-2. https://doi.org/10.1097/jxx.0000000000000373.

58. Terry AB, Wylie A, Raspa M, et al. Clinical models of telehealth in genetics: A regional telegenetics landscape. J Genet Couns. 2019;28(3):673-91. https://doi.org/10.1002/jgc4.1088.

59. Solomons NM, Lamb AE, Lucas FL, McDonald EF, Miesfeldt S. Examination of the patient-focused impact of cancer telegenetics among a rural population: comparison with traditional in-person services. Telemed J E Health. 2018;24(2):130-8. https://doi.org/ 10.1089/tmj.2017.0073.

60. Shannon KM, Emmet MM, Rodgers LH, Wooters M, Seidel ML. Transition to telephone genetic counseling services during the COVID-19 pandemic. J Genet Couns. 2021;30(4):984-8. https:// doi.org/10.1002/jgc4.1365.

61. McDonald E, Lamb A, Grillo B, Lucas L, Miesfeldt S. Acceptibility of telemedicine and other cancer genetic counseling models of service delivery in geographically remote settings. J Genet Couns. 2014;23(2):221-8. https://doi.org/10.1007/s10897-013-9652-9.

62. McCuaig JM, Randall Armel S, Care M, Volenik A, Kim RH, Metcalfe KA. Next-generation service delivery: a scoping review of patient outcomes associated with alternative models of genetic counseling and genetic testing for hereditary cancer. Cancers. 2018;10(11):435. https://doi.org/10.3390/cancers10110435.

63. Samimi G, Bernardini MQ, Brody LC, et al. Traceback: a proposed framework to increase identification and genetic counseling of BRCA1 and BRCA2 mutation carriers through family-based outreach. J Clin Oncol. 2017;35(20):2339-2337. https://doi.org/ 10.1200/jco.2016.70.3439.

64. Nelson HD, Pappas M, Cantor A, et al. Risk assessment, genetic counseling, and genetic testing for BRCA-related cancer in women: updated evidence report and systematic review for the US Preventive Services Task Force. JAMA. 2019;322(7):666-85. https://doi.org/10.1001/jama.2019.8430.

65. Dean M, Boland J, Yeager M, et al. Addressing health disparities in Hispanic breast cancer: accurate and inexpensive sequencing of BRCA1 and BRCA2. Gigascience. 2015;4(4):50. https://doi. org/10.1186/s13742-015-0088-z. 
66. Peterson SK. The role of the family in genetic testing: theoretical perspectives, current knowledge, and future directions. Health Educ Behav. 2005;32(5):627-39. https://doi.org/10.1177/10901 98105278751.

67. Forbes Shepherd R, Browne TK, Warwick L. A relational approach to genetic counseling for hereditary breast and ovarian cancer. J Genet Couns. 2017;26(2):283-99. https://doi.org/10. 1007/s10897-016-0022-2.

68. Armstrong K, Micco E, Carney A, Stopfer J, Putt M. Racial differences in the use of BRCA $1 / 2$ testing among women with a family history of breast or ovarian cancer. JAMA. 2005;293(14):172936. https://doi.org/10.1001/jama.293.14.1729.

69. Hawkins AK, Hayden MR. A grand challenge: Providing benefits of clinical genetics to those in need. Genet Med. 2011;13(3):197200. https://doi.org/10.1097/gim.0b013e31820c056e.

70. Fehniger J, Lin F, Beattie MS, Joseph G, Kaplan C. Family communication of BRCA $1 / 2$ results and family uptake of BRCA $1 / 2$ testing in a diverse population of BRCA $1 / 2$ carriers.
J Genet Couns. 2013;22(5):603-12. https://doi.org/10.1007/ s10897-013-9592-4.

71. Trottier M, Lunn J, Butler R, et al. Strategies for recruitment of relatives of BRCA mutation carriers to a genetic testing program in the Bahamas. Clin Genet. 2015;88(2):182-6. https://doi.org/ $10.1111 /$ cge. 12468 .

72. Houlihan MC, Tariman JD. Comparison of outcome measures for traditional and online support groups for breast cancer patients: an integrative literature review. J Adv Pract Oncol. 2017; 8(4): 348-359. https://www.ncbi.nlm.nih.gov/pmc/articles/PMC60 $40879 /$.

Publisher's Note Springer Nature remains neutral with regard to jurisdictional claims in published maps and institutional affiliations. 